Judith Peters*, Nicolas Martinez, Grégoire Michoud, Anaïs Cario, Bruno Franzetti, Philippe Oger, and Mohamed Jebbar

\title{
Deep Sea Microbes Probed by Incoherent Neutron Scattering Under High Hydrostatic Pressure
}

\begin{abstract}
The majority of the biosphere is a high pressure environment. Around $70 \%$ of the marine biosphere lies at depths below $1000 \mathrm{~m}$, i.e. at pressures of 100 bars or higher. To survive in these environments, deep-biosphere organisms have adapted to life at high pressure. In vitro studies showed that the activity of certain proteins originating from deep-sea organisms is less affected by high pressure than that of enzymes from surface organisms [1-3]. However, the genetic and structural bases for this increased pressure resistance are still unknown. Elastic incoherent neutron scattering studies, which provide access to information about molecular dynamics, constitute a very promising approach to decipher the structural adaptation in proteins living under high pressure. This approach has been used in the past to investigate the adaptation of biological systems to temperature and salinity and proved to be essential and complementary to structural studies. Here first investigations of high pressure effects on cell dynamics are presented using Thermococcales as models.
\end{abstract}

Keywords: Neutron Scattering, Molecular Dynamics, High Pressure, Deep Sea Microbes.

DOI 10.1515/zpch-2014-0547

Received June 03, 2014; accepted July 08, 2014

\footnotetext{
*Corresponding Author: Judith Peters, Institut Laue Langevin, F-38042 Grenoble Cedex 9, France, e-mail: peters@ill.fr

Nicolas Martinez: Institut Laue Langevin, F-38042 Grenoble Cedex 9, France Judith Peters, Nicolas Martinez, Bruno Franzetti: Institut de Biologie Structurale (IBS), CNRS/CEA/Grenoble-Alpes Univ., F-38042 Grenoble Cedex 9, France Judith Peters, Nicolas Martinez: Université Joseph Fourier, F-38041 Grenoble Cedex 9, France Grégoire Michoud, Mohamed Jebbar: Laboratoire de Microbiologie des Environnements Extrêmes, UMR 6197 UBO CNRS Ifremer, IUEM Technopole Brest-Iroise, F-29280 Plouzane, France

Anaïs Cario, Philippe Oger: Laboratoire de Géologie de Lyon, UMR CNRS 5276, Ecole Normale Supérieure de Lyon, F-69364 LYON cedex 07, France
} 


\section{Introduction}

Recent estimations suggest that life on Earth dwells mostly in the so-called "DeepBiosphere", e.g. below the surface of the Earth. The deep biosphere, located in the continental underground and in the oceans below $1000 \mathrm{~m}$ in depth, could represent up to $70 \%$ of all cells on Earth, as well as $50 \%$ of the primary production of biomass. Although the deep biosphere represents therefore the largest ecosystem on Earth, it is still poorly characterized in terms of diversity or function $[4,5]$. Most of these biotopes are oligotrophic in nature, and characterized by high hydrostatic pressures (HHP). HHP is inhibitory to many biological functions, and deep-biosphere organisms have developed adaptive strategies to counteract its effects, but their mechanism remains largely unknown so far.

Several HHP-adapted organisms, or piezophiles (from the greek piezo = to press and philo = love, piezophiles have optimal growth rates at pressures greater than 1 atmosphere or $0.1 \mathrm{MPa}$ ), have been isolated, such as in 1981 the strain MT41 of Colwellia, the first obligate piezophile organism [6], and in 2009 the Pyrococcus yayanosii strain $\mathrm{CH} 1$, the first obligate piezophile and hyperthermophile isolated from the Ashadze site. These organisms require HHP for growth, and have pressure optima in excess of the permissive pressure for surface organisms. Our current knowledge clearly supports the existence of an adaptation to HHP in piezophilic organisms. Indeed HHP strongly impacts the cell membrane and the cellular proteins. HHP will affect cells and cellular components according to Le Chatelier's principle. In brief, increasing pressure will favor the state of lower volume. For example, HHP will increase the packing of lipids which will reduce their biological activity $[7,8]$. Similarly, HHP will tend to destabilize protein multimeric edifices and proteins, with multiple dramatic consequences on cellular functions. If it is well established that microorganisms adapt to HHP, the putative adaptation strategies are still elusive. Three different strategies have been proposed, which require 1) the fine tuning of gene regulation to compensate for the pressure-dependent loss of activity of proteins, or 2) the expression of HHPspecific genes or 3) the existence of structural adaptation in proteins [9]. For instance, it is now well documented that microorganisms adapt the lipid composition of their membranes in order to maintain an appropriate permeability and viscosity, a phenomenon referred to as homeoviscous adaptation [10] to counteract the rigidifying effect of high pressure and maintain membranes in the physiological liquid state cells by incorporating lipids with increased unsaturation levels. The kinked chain of unsaturated lipids decreases the chain ordering and thus lowers the transition temperature $T_{m}$ [11] helping in this way the organisms to survive 
under high pressure conditions. Yet it is unclear how proteins from piezophilic organisms have adapted.

Elastic incoherent neutron scattering (EINS) experiments have recently revealed important differences in the mean molecular dynamics between organisms adapted to high and low temperature habitats [12]. The mean flexibility and resilience of the macromolecules were obtained and gave valuable insights on how thermoadaptation in macromolecules is achieved. These experiments were performed on whole living cells thus allowing studying the dynamics of biomolecules in their cellular context. The signal obtained from elastic neutron scattering experiments on whole cells is in fact expected to be dominated by the contribution from the proteins [12]. In adaptation studies, measuring whole cell systems is therefore advantageous compared to experiments on individual proteins as the adaptation mechanism of one specific protein can deviate significantly from the predominant mechanism.

\section{Materials and methods}

\subsection{Sample preparation}

Microorganisms used in this study were Thermococcus barophilus, a piezophile and hyperthermophile, which grows optimally at $40 \mathrm{MPa}$ and $85^{\circ} \mathrm{C}$ and Thermococcus kodakarensis, a hyperthermophile, which lives at atmospheric pressure and $85^{\circ} \mathrm{C}$. Escherichia coli was used as a mesophile reference. Cells of T. barophilus and T. kodakarensis were cultivated in TRM medium [13] at atmospheric pressure and $85^{\circ} \mathrm{C}$ until late exponential phase at the LM2E laboratory in Brest (France). Under anoxic conditions, cells were washed once in isotonic solution, pelleted in a high pressure aluminum capsule, frozen at $-80{ }^{\circ} \mathrm{C}$ and transferred in dry ice to the Institut Laue Langevin (ILL) in Grenoble (France), in order to avoid damages due to oxygen contamination during transport.

For the neutron experiments the thin aluminum cartridges of a diameter of $2 \mathrm{~mm}$ and a height of $3 \mathrm{~cm}$ were loaded into the high pressure container under anaerobic conditions at room temperature to limit the contact of the samples with oxygen. The sample volume (6 mm diameter) was further filled with $\mathrm{D}_{2} \mathrm{O}$ as a pressure transmission medium (see Figure 1). As neutrons are incoherently scattered essentially by hydrogen atoms (see Section 2.3 below), this allows to probe mainly the cells and the $\mathrm{H}_{2} \mathrm{O}$ molecules inside the cells or bound to their surface. Under the experimental conditions, the samples were stable for several days at atmospheric pressure. 


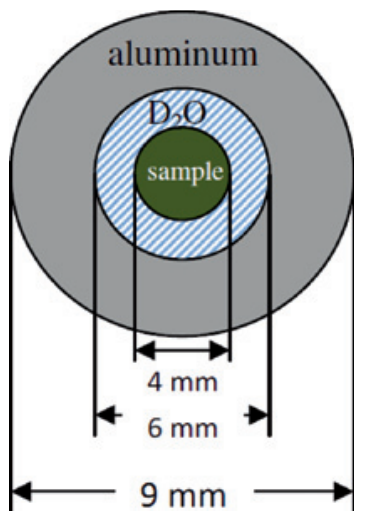

Figure 1: View from the top of the experimental setup of the cylindrical high pressure container. The container is built of a high-tensile aluminum alloy; it is filled with an aluminum cartridge containing the sample and with $\mathrm{D}_{2} \mathrm{O}$.

\subsection{High pressure equipment}

A high pressure equipment dedicated to neutron scattering experiments of biological samples in solution has been developed over the last years at the ILL in Grenoble [14]. In brief, it consists of a high pressure stick, which can be placed in the cryostats or cryofurnaces of the instruments, a pressure controller which allows a remote control and permanent adjustment of pressure and cylindrical pressure containers built of a high-tensile aluminum alloy. Two high pressure sample holders are currently available, withstanding pressures up to $7 \mathrm{kbar}(700 \mathrm{MPa})$ and $1.5 \mathrm{kbar}$, respectively. The latter one was used in the experiments presented here.

\subsection{Neutron scattering experiments}

Whereas coherent neutron scattering takes advantage of the interference of neutron waves scattered from different atomic nuclei preserving the phase, incoherent scattering sees neutron waves scattered by one and the same nucleus at different times, which gives rise to a self-diffusion effect [15]. Spectrometers allow to investigate incoherent neutron scattering by using samples in solution, what destroys spatial ordering, to follow averaged atomic motion of individual particles in time. The incoherent neutron scattering intensity is dominated by the signal arising from hydrogen. This is due to the hydrogen incoherent scattering cross section, which is one order of magnitude larger than that of all other elements usually oc- 
curring in biological matter, and also of its isotope deuterium [16]. The technique thus probes average protein dynamics, because hydrogen atoms are almost uniformly distributed in the protein and representative for the molecular subgroups to which they are bound. Thus when using $\mathrm{D}_{2} \mathrm{O}$ for the surrounding water, its signal is negligible compared to signal arising from the sample itself and the light water contained in it (the contribution can be estimated to be less than $2 \%$ ).

For the actual study we used a backscattering spectrometer, IN13 [17], of the ILL in Grenoble/France. It has an energy resolution of $8 \mu \mathrm{eV}$, corresponding to a time window up to 100 ps. It is therefore very well suited for such studies as it permits to see internal motions, arising mainly from the amino acid side chains, without mixing them with a signal from global diffusive motions or water motion. The latter movements correspond in fact to longer and smaller time scales, respectively. In consequence, a spectrometer acts as a motion filter highlighting specific dynamics in the samples. Elastic scattering data, corresponding to the situation where no energy is exchanged, but the neutrons are deviated from their initial direction giving rise to an exchange of momentum, designated as $Q$, were analyzed through the scattering function $S(Q, 0 \pm \Delta E)$, where $\Delta E$ means the instrumental resolution. Assuming a normal distribution of the atoms around their average position it reduces in terms of the Gaussian approximation [18] to:

$$
S(Q, 0 \pm \Delta E) \approx \exp \left(-\frac{1}{6} Q^{2}\left\langle u^{2}\right\rangle\right)
$$

Here $\left\langle u^{2}\right\rangle$ are the average atomic mean-square displacements (MSD). The MSD values are obtained for each temperature/pressure point from the slope of the logarithm of the scattered intensities $S(Q, 0 \pm \Delta E)$ plotted versus $Q^{2}$ according to

$$
\left\langle u^{2}\right\rangle=-6 \frac{\partial \ln S(Q, 0 \pm \Delta E)}{\partial Q^{2}} .
$$

This approximation is strictly speaking only valid for $Q \rightarrow 0$, but can be extended to $\left\langle u^{2}\right\rangle Q^{2}<2$ [19]. Accordingly, in this study the fit range was restricted to the linear range in the low $Q$ regime between $0.5 \AA^{-1}$ and $1.9 \AA^{-1}$.

Each pressure and temperature point was measured for at least $6 \mathrm{~h}$ to ensure a sufficient signal-to-noise ratio. For correction and normalization purposes, the empty cell, the buffer $\left(\mathrm{D}_{2} \mathrm{O}\right)$ and a vanadium rod (a completely incoherent scatterer) were recorded under similar conditions. Absorption corrections based on the formula of Paalman-Pings [20] were applied and the complete data reduction was carried out using the LAMP software available at the Institut Laue Langevin (ILL) $[21,22]$. 


\section{Results and discussion}

\subsection{Summed neutron intensities}

The main challenge in such experiments arises from the fact that prokaryotic cells are highly complex biological systems. They are composed of several compartments (membrane, cytoplasm, skeleton, ribosomes), which are composed of lipids, cholesterol, proteins, nucleic acids, water, and numerous other molecules. Thus reproducibility of the experiments may be an issue, even when cells are prepared with the greatest care [23, 24]. Neutron scattering experiments are long (typically several days) and usually require the isolation of the cells from nutriments or the use of physico-chemical conditions far from the cell optima. Moreover, the high pressure container absorbs the neutrons much more than standard neutron scattering sample holders, therefore the statistics are poor, further increasing acquisition time. The current series of measurements totaled 3 weeks data acquisition over a 3-year period. In these conditions, it is crucial to determine the most appropriate handling procedures for each type of cells to prepare them for the measurements.

Figures 2-4 show the collected intensities, corrected for the empty cell and buffer contributions and normalized to vanadium, summed over a $Q$-range from 0.5 to $2.1 \AA^{-1}$. Starting from the Gaussian approximation Equation (1), one shows that the intensities summed over a limited Q-range are inversely proportional to the square root of the MSD. However, the sum over the intensities does not require the Gaussian approximation, so that such approach permits to use a broader Qrange and a fast evaluation of the runs with higher statistics. The absolute height of the summed intensities depends mainly on the sample mass in the neutron beam. The curves were therefore normalized to allow the direct comparison of their slopes.

At a first glance, all three samples clearly show an increase of the intensities with pressure. As the pressure dependence of the intensities is expected to be inversely related to the behavior of the MSD, such behavior could be expected in agreement with Le Chatelier's principle: high pressure favors a decrease in volume and thus a damping of atomic motions. It is noteworthy that the results are extremely similar for the independent measurements performed over the three years. The error bars take into account statistical uncertainties of the data, but cannot represent differences in the experimental conditions. Taking into account the difficulties inherent to neutron experimentation with samples in a high pressure and anaerobic environment, the agreement is thus rather satisfying. 


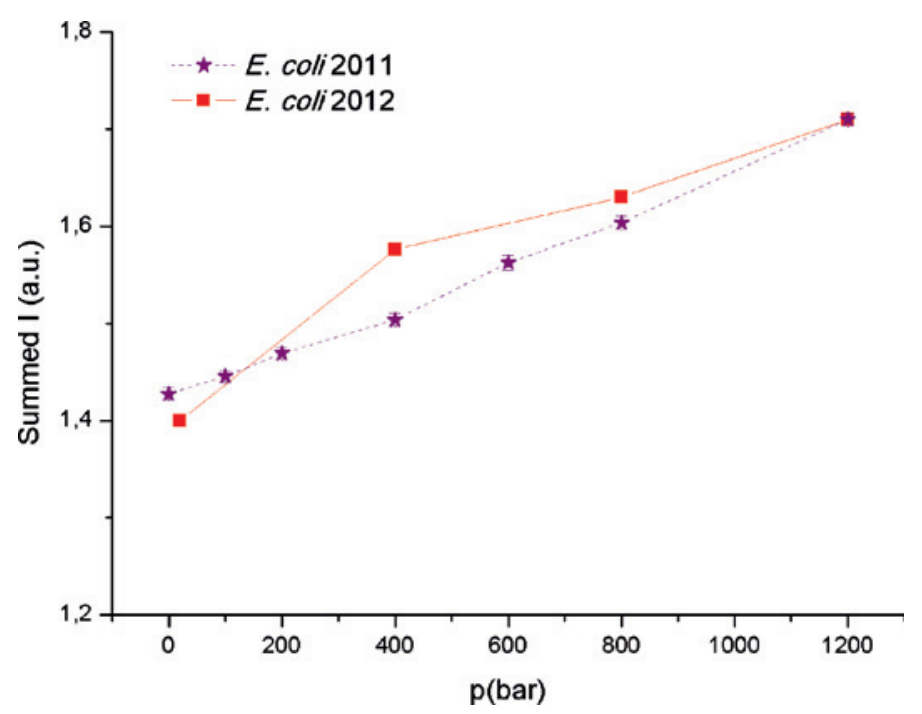

Figure 2: Neutron intensities summed over the $Q$-range from 0.5 to $2.1 \AA^{-1}$ for all three samples: represents data for $E$. coli. The stars and dashed lines represent results from 2011, the filled symbols represent results from 2012.

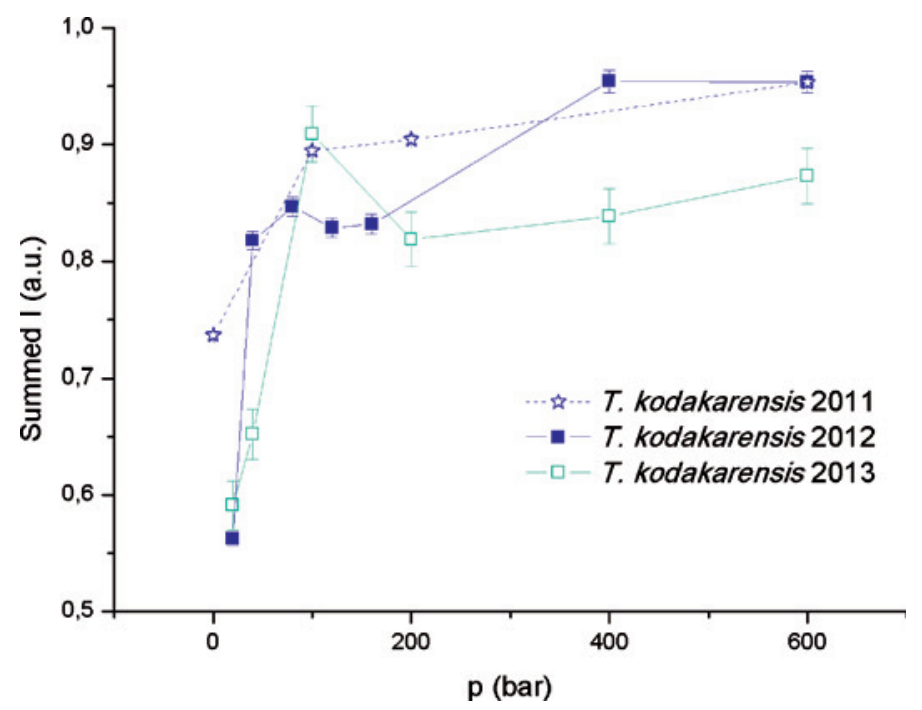

Figure 3: Neutron intensities summed over the $Q$-range from 0.5 to $2.1 \AA^{-1}$ for all three samples: represents data for $T$. kodakarensis. The stars and dashed lines represent results from 2011, the filled symbols represent results from 2012 and the open symbols from 2013. 


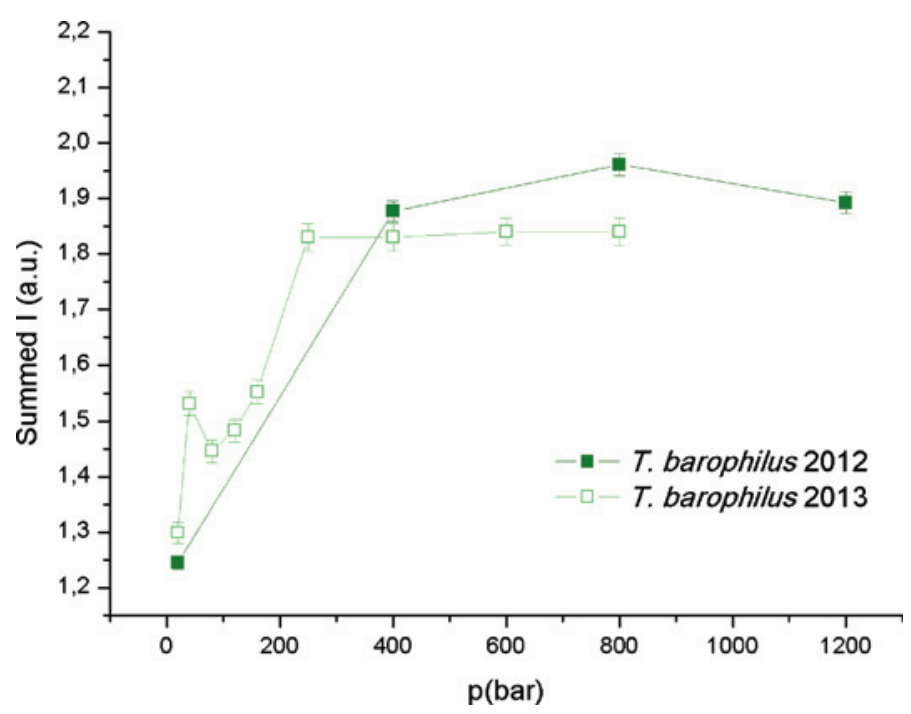

Figure 4: Neutron intensities summed over the Q-range from 0.5 to $2.1 \AA^{-1}$ for all three samples: represents data for T. barophilus. The filled symbols represent results from 2012 and the open symbols from 2013.

The intensities' increase with pressure differs from one sample to the other: whereas it looks mostly linear for E. coli, the curves for T. kodakarensis and T. barophilus show two drastically different regimes, with a rapid increase, followed by a plateau. Interestingly, this jump occurs at much lower pressures for the piezosensitive T. kodakarensis, ca. $30 \mathrm{bar}$, than for the piezophile $T$. barophilus, ca. 200 bar. Moreover, the relative increase in summed intensities after the jump seemed to be higher for T. kodakarensis than for T. barophilus, which may indicate that the T. barophilus cells are less affected by HHP than the T. kodakarensis cells. These observations are in agreement with the strain physiology; e.g. sensitivity to HHP in T. kodakarensis and piezophily for T. barophilus.

The MSD - and therefore the inverse of the summed intensities - stand for the flexibility of the sample under specific conditions in pressure and temperature. Comparative measurements of samples under identical conditions permit thus to conclude about their flexibility in a given environment. Moreover, it was possible in the past $[25,26]$ to establish a function-structure-dynamics relationship showing that proteins can only be operational when they are in a folded, thus native, state, in presence of sufficient hydration and having acquired an adequate flexibility. Indeed when scanning proteins over a large temperature range, going from non-physiological states at $20 \mathrm{~K}$ to ambient temperature, a significant change in the mean square displacements occurs typically around $220 \mathrm{~K}$. In the literature it 
is often referred as "dynamical transition" and interpreted in terms of a transition from purely harmonic motions to anharmonic movements. Obviously the latter ones are mandatory for the functioning of the protein.

It is, however, still unknown if a similar transition exists as function of high pressure. Not many experimental data are available today for proteins exposed to HHP [27-29] and only a few simulation predictions [30, 31]. They show that a reduction of dynamics with increasing pressure is expected, corresponding first to a reduction of the cavities' volumes, than to a partial unfolding giving access to some internal cavities to water molecules and finally to a complete unfolding process. Protein denaturation induced by unfolding normally occurs at higher pressure than those tested in this experiment, typically above several kbar. In the present case, the results obtained for $E$. coli most probably reflect a linear decrease in flexibility with increasing pressure. However, such a pressure-dependent decrease in flexibility cannot explain the sudden decrease in molecular dynamics observed for the two thermophilic strains T. kodakarensis and T. barophilus. Their dynamics' decrease is likely to be correlated with structural re-arrangements of macromolecules within the cells. Although our data does not allow to decide which molecules are responding to pressure, there are two major molecules types that are likely to be affected by hydrostatic pressure in this pressure range: the lipids from the membranes and the cell proteins. Membranes are one of the most pressure sensitive compartments of the cell [32]. Pressure variations permit to shift their phase transition temperatures and therefore the structural re-arrangements, which is visible in the dynamics determined through MSD measurements [33]. Several cellular proteins, such as actin, which cortical organization is disrupted by increasing HHP, are also pressure sensitive [34]. Increasing HHP also strongly impact the size and shape of cavities inside proteins which will lead to further structural rearrangements [35].

At a certain pressure, which depends on the individual strain, a structural optimum is reached, and allows to withstand high pressure. It is interesting to note that the pressure at the jump for T. barophilus and T. kodakarensis are close to their optimal growth pressures. Once the optimum state of stability is reached, another regime starts where the summed intensities increase slightly for T. kodakarensis with a slope similar to that observed for $E$. coli, which indicate the reduction of protein flexibility for these organisms with pressure. Despite their different temperature optima, e.g. $37^{\circ} \mathrm{C}$ and $85^{\circ} \mathrm{C}$ for E. coli and T. kodakarensis, respectively, we observed a decrease of the dynamics with increasing pressure for both organisms, which is consistent with the fact that both organisms are sensitive to hydrostatic pressure. In contrast to T. kodakarensis, T. barophilus is piezophilic, and has an optimal growth pressure at ca. 400 bar. It is striking that the summed intensities for T. barophilus remain constant from that pressure up to at least $1.2 \mathrm{kbar}$, 


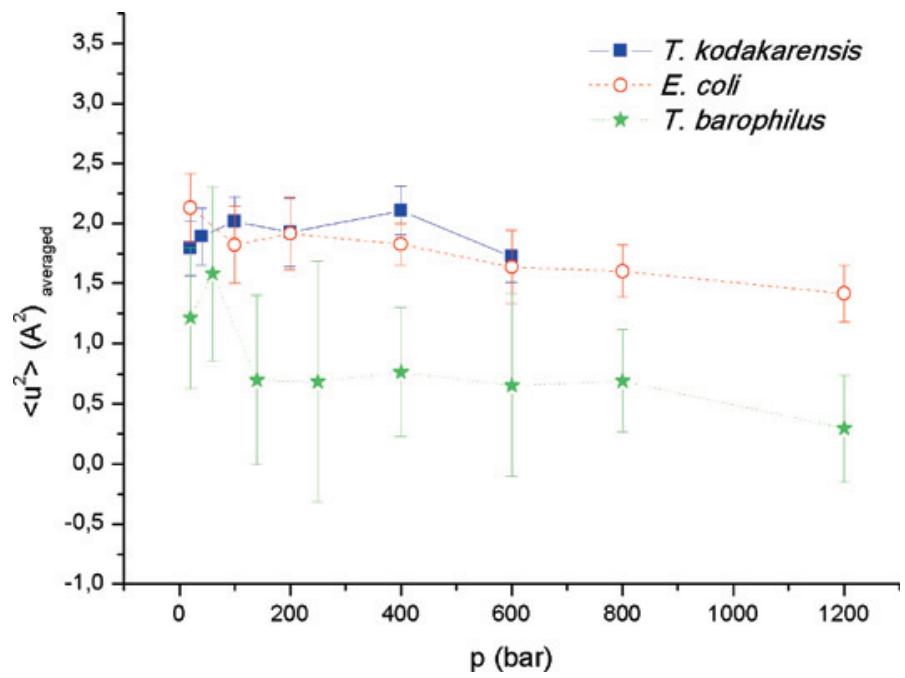

Figure 5: MSD extracted from the measurements performed in 2012 and 2013 and averaged after checking their reproducibility.

indicating the lack of a pressure-dependent loss of protein flexibility in this organism. Although it does not tell us which molecule/structure is affected, this clearly shows that the cell dynamics is different between pressure adapted and pressure sensitive organisms.

\subsection{Atomic mean square displacements}

To go further in data analysis, we extracted the MSD (Figure 5). Data from 2012 and 2013 were averaged to increase the counting statistics.

The trend is confirmed that the MSD decrease with pressure as predicted by Le Chatelier's principle. The error bars are rather high, but the data permit to present first results for such complex systems under high pressure. Similarly to what is observed for the summed intensities, the pressure-dependence of the MSD for E. coli is almost linear. For T. kodakarensis and T. barophilus some fluctuations are visible at low pressure reflecting the jump-like behavior described above. The relative decrease of the MSD between 20 and 1200 bar depends on the sample, but lies around $30 \%-40 \%$ for all three (when extrapolating the value for T. kodakarensis at 1200 bar and neglecting the first two points for T. barophilus, which are associated to the jump). Typical compressibilities of globular proteins in solution would give rise to linear compression of about $3 \%$, only [36], therefore compressibility is 
probably not the solely responsible for the observed effect. Rather there must be additional structural re-arrangements, as already suggested above.

The main difficulty concerning the interpretation of these results arises from the fact that $T$. kodakarensis and T. barophilus originate from a high temperature environment. Both live close to hot vents at about $85^{\circ} \mathrm{C}$. However, the high-tensile aluminum alloy (7049-T6) used to build up the high pressure container does not withstand temperatures higher than $50{ }^{\circ} \mathrm{C}$, the material becomes then brittle and breaks. Therefore, the measurements were done at room temperature, which is far from the native conditions of these cells. In consequence, it is not clear how representative these results are with respect to the dynamics of the cells under growth permissive conditions. It should be reminded that the above mentioned dynamical transition occurs at $220 \mathrm{~K}$ which is as far from physiological temperatures for proteins. After this point, the slope stays constant up to at least ambient conditions. As a consequence, it may be possible to extract information on the flexibility state of proteins under physiological conditions knowing their behavior at lower temperatures by a linear extrapolation. This behavior has been observed and exploited by Tehei et al. [9] to estimate the MSD of thermo- and hyperthermophile bacteria under their environment growth temperatures, which could not be reached during the experiment. If the generality of such a linear increase of the MSD cannot be proven easily, inversely it cannot be excluded, so that our results at lower temperature might indeed be representative for higher temperature. Additional experiments would be required to confirm this hypothesis.

\section{Conclusions}

EINS experiments were performed on whole cells of different bacteria and archaea from the Thermococcales under hydrostatic pressure conditions up to $1.2 \mathrm{kbar}$. It is a potent method to characterize molecular dynamics and to shed light on adaptation mechanisms to an extreme environmental condition such as high pressure. However, combining neutron scattering and high pressure is an extremely demanding task, the more as the samples had to be only handled under anaerobic conditions. The experiments were repeated three consecutive years at the ILL in Grenoble/France and the intensities summed over a certain scattering angle range show a rather good reproducibility.

Some differences in summed intensities between the probed systems were identified: The reference mesophile system $E$. coli had a linear behavior with increasing pressure, whereas the hyperthermophile T. kodakarensis and the piezophile and hyperthermophile $T$. barophilus underwent a jump-like increase 
in the intensities around 30 and 200 bar, respectively. The extracted MSD have large error bars, but indicate dynamical changes and structural re-arrangements at low pressure of the Thermococcales up to an optimum pressure followed by a much higher stability above it. It is interesting to note that the slope for the two piezosensitive organisms display similar decrease in molecular flexibility despite their temperature optima, while HHP seem to have a limited impact on the piezophilic strain. These observations would need further investigations, since the experiments could not be performed at native temperatures, ca. $85^{\circ} \mathrm{C}$, for T. kodakarensis and T. barophilus, as the high pressure cell is not withstanding high temperatures. We cannot exclude that the slopes for molecular dynamics under HHP in the thermophilic strains could be similar under physiological temperatures. A new high pressure cell allowing to reach higher temperatures is actually under development at the ILL to perfom such investigations. Nevertheless and in light of the extreme complexity and difficulties of the investigations, it seems possible to conduct such studies in vivo through incoherent neutron scattering.

Acknowledgement: This work was supported by grants from the Project ANR 2010 BLAN 172501 Living deep. We gratefully acknowledge Giuseppe Zaccaï for fruitful discussions. Finally, we thank Mathieu Lemé and all members of the SANE group from the ILL (Jean-Luc Laborier, Claude Payre, Jean-Paul Gonzales, Simon Baudoin, Nadir Belkhier and Eddy Lelièvre-Berna) for their help during the development of the high-pressure equipment and the ILL for according the beam time.

\section{References}

1. A. A. Brindley, R. W. Pickersgill, J. C. Partridge, D. J. Dunstan, D. M. Hunt, and M. J. Warren, PLOS ONE 3 (2008) e2042.

2. T. Morita, J. Biol. Chem. 278 (2003) 28060.

3. Y. Nishiguchi, T. Miwa, and F. Abe, Extremophiles 12 (2008) 477.

4. W. B. Whitman, D. C. Coleman, and W. J. Wiebe, P. Natl. Acad. Sci. USA 95 (1998) 6578.

5. J. Kallmeyer, R. Pockalny, R. R. Adhikari, D. C. Smith, and S. D’Hondt, P. Natl. Acad. Sci. USA 109 (2012) 16213.

6. A. A. Yayanos, A. S. Dietz, and R. Van Boxtel, P. Natl. Acad. Sci. USA 78 (1981) 5212.

7. C. Balny, J. Phys.-Condens. Mat. 16 (2004) S1245.

8. F. Pradillon and F. Gaill, Rev. Environ. Sci. Biotechnol. 6 (2007) 181.

9. P. Oger and M. Jebbar, Res. Microbiol. 161 (2010) 799.

10. M. Sinensky, P. Natl. Acad. Sci. USA 71 (1974) 522.

11. D. H. Bartlett, Biochim. Biophys. Acta 1595 (2002) 367.

12. M. Tehei, B. Franzetti, D. Madern, M. Ginzburg, B.Z. Ginzburg, M. T. Giudici-Orticoni, M. Bruschi, and G. Zaccai, EMBO Rep. 5 (2004) 66. 
13. S. Zeng, J. Birrien, Y. Fouquet, G. Cherkashov, M. Jebbar, J. Querellou, P. Oger, M.-

A. Cambon-Bonavita, X. Xiang, and D. Prieur, ISME J. 3 (2009) 873.

14. J. Peters, M. Trapp, D. Hughes, S. Rowe, B. Demé, J.-L. Laborier, C. Payre, J.-P. Gonzales, S. Baudoin, N. Belkhier, and E. Lelievre-Berna, High Pressure Res. 32 (2011) 97.

15. Doster W., H. Nakagawa, and M. S. Appavou , J. Chem. Phys. 139 (2013) 045105.

16. V. F. Sears, Neutron News 3 (1992) 26.

17. F. Natali, J. Peters, D. Russo, S. Barbieri, C. Chiapponi, A. Cupane, A. Deriu, M. T. Di Bari, E. Farhi, Y. Gerelli, P. Mariani, A. Paciaroni, C. Rivessau, G. Schiro', and F. Sonvico, Neutron News 19 (2008) 14.

18. A. Rahman, K. S. Singwi, and A. Sjölander, Phys. Rev. 126 (1962) 986.

19. V. Réat, G. Zaccai, C. Ferrand, and C. Pfister, Functional Dynamics in Purple Membranes, in: Biological Macromolecular Dynamics, S. Cusack, H. Buttner, M. Ferrand, P. Langan, P. Timmins (Eds.), Adenine Press, Schenectady, NY (1997), pp. 117-122.

20. H. H. Paalman and C. J. Pings, J. Appl. Phys. 33 (1962) 2635.

21. D. Richard, M. Ferrand, and G. J. Kearley, J. Neutron Res. 4 (1996) 33.

22. LAMP, the Large Array Manipulation Program, http://www.ill.eu/data_treat/lamp/ the-lamp-book/.

23. V. Marty, M. Jasnin, E. Fabiani, P. Vauclare, F. Gabel, M. Trapp, J. Peters, G. Zaccai, and B. Franzetti, J. R. Soc. Interface 10 (2013) 20130003.

24. M. Tehei, B. Franzetti, K. Wood, F. Gabel, E. Fabiani, M. Jasnin, M. Zamponi, D. Oesterhelt, G. Zaccai, M. Ginzburg, and B. Z. Ginzburg, P. Natl. Acad. Sci. USA 104 (2007) 766.

25. W. Doster, S. Cusack, and W. Petry, Nature 337 (1989) 754.

26. G. Zaccai, Science 288 (2000) 1604.

27. A. Filabozzi, A. Deriu, M. T. Di Bari, D. Russo, S. Croci, and A. Di Venere, Biochim. Biophys. Acta, Proteins Proteom., 1804 (2010) 63.

28. M. G. Ortore, F. Spinozzi, P. Mariani, A. Paciaroni, L. R. S. Barbosa, H. Amenitsch, M. Steinhart, J. Ollivier, and D. Russo, J. R. Soc. Interface 6 (2009) 619.

29. W. Doster and R. Gebhardt, Chem. Phys. 292 (2003) 383.

30. L. Meinhold and J. C. Smith, Phys. Rev. E 72 (2005) 061908.

31. L. Meinhold, J. C. Smith, A. Kitao, and A. H. Zewail, P. Natl. Acad. Sci. USA 104 (2007) 17261.

32. G. N. Somero, Annu. Rev. Physiol. 54 (1992) 557.

33. M. Trapp, J. Marion, M. Tehei, B. Demé, T. Gutberlet, and J. Peters, Phys. Chem. Chem. Phys. 15 (2013) 20951.

34. D. A. Begg, E. D. Salmon, and H. A. Hyatt, J. Cell Biol. 97 (1983) 1795.

35. E. Girard, R. Kahn, M. Mezouar, A. C. Dhaussy, T. Lin, J. E. Johnson, and R. Fourme, Biophys. J. 88 (2005) 3562.

36. K. Gekko and H. Noguchi, J. Phys. Chem. 83 (1979) 2706. 